\title{
EINE LESEHILFE FÜR DIESES BUCH
}

Dieses Buch ist auf drei Ebenen geschrieben. Das Neue, was uns in den Kopf gekommen ist, das bieten wir ihnen ganz herkömmlich an: Sie lesen unsere Texte in ihrer linearen Darstellungsweise und können hoffentlich unseren Gedanken folgen, warum wir so denken, handeln, forschen und experimentieren. Wie es sich auf der linearen Ebene gehört, wollen die Gedanken hier auch nachvollziehbar zu einem logischen Ende gebracht werden.

Auf einer anderen Ebene, die wir die 3D-Ebene nennen wollen, bieten wir Ihnen die Bilder unserer Aufstellungen an. Hier agieren wir mit Optionen, mit mutigen Annahmen und mit ungewöhnlichen Bildern und es ist an Ihnen, das Neue einzuladen. Lassen Sie sich irritieren, überwinden Sie den ersten inneren Impuls, das Andere abzulehnen und achten Sie auf die Bilder und Vermutungen, die in Ihrem Kopf auftauchen.

Auf dieser bildlichen Ebene der Darstellung komplexer Systeme darf und soll sich bei Ihnen sich das Neue zeigen. Es sind zumeist Erkenntnisse, die genau in der Lücke der Irritation entstehen und plötzlich sehen Sie Zusammenhänge, die Sie zuvor nicht gesehen haben. Komplexität lässt sich manchmal auch intuitiv erfassen und genau dazu wollen wir Sie einladen. Reagieren Sie nicht nur mit dem Kopf auf rationaler Ebene, sondern mit dem ganzen Körper, hören Sie ihm zu und beobachten sie die Veränderungen.

Wir können Ihnen nicht versprechen, dass neue Bilder entstehen und noch viel weniger können wir vorhersagen, welche Bilder entstehen. Halten Sie sich mutig im Raum der Irritationen auf und spüren das Neue in Ihnen. Und dann kommen Sie wieder zurück auf die erste Ebene und folgen unseren linearen Texten. Oder wenn es Ihnen zu gut gefällt: Dann wandern Sie nur noch von Systembild zu Systembild durch das Buch.

Welche Verbindungen zwischen Menschen entstehen, die mithilfe von Aufstellungen lehren und forschen, wollen wir ihnen auf einer dritten Ebene zeigen. Wir lassen einige Menschen, die uns in den letzten Jahren begleitet und dabei geholfen haben, Zuversicht in eine faszinierende Methode zu gewinnen, zu Wort kommen und ihre Eindrücke skizzieren. Lassen Sie sich von Selbstbeobachtungen dazu animieren, auch selbst eigene Erfahrungen mit Systemaufstellungen zu machen.

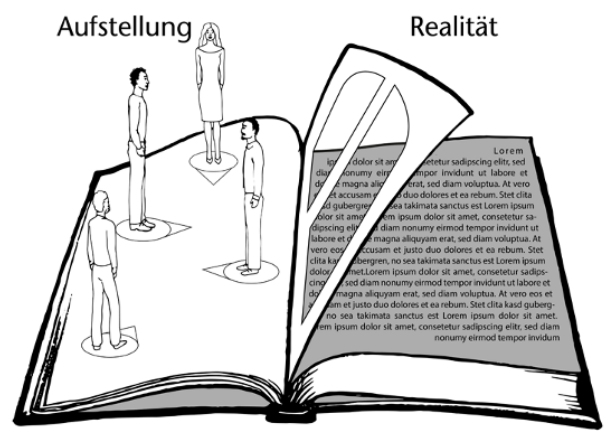

